\title{
Limited effect of screening for depression with written feedback in outpatients with diabetes mellitus: a randomised controlled trial
}

\author{
F. Pouwer • C. J. Tack • \\ P. H. L. M. Geelhoed-Duijvestijn • E. Bazelmans • \\ A. T. Beekman • R. J. Heine • F. J. Snoek
}

Received: 9 June 2010 / Accepted: 6 December 2010 /Published online: 11 January 2011

(C) The Author(s) 2011. This article is published with open access at Springerlink.com

\begin{abstract}
Aims/hypothesis The aim of this study was to test the effectiveness of a screening procedure for depression (SCR) vs care as usual (CAU) in outpatients with diabetes. The primary outcome measured was depression score and the secondary outcomes were mental healthcare consumption, diabetes-distress and $\mathrm{HbA}_{1 \mathrm{c}}$.

Materials and methods In a multicentre parallel randomised controlled trial, 223 outpatients with diabetes, who had an elevated depression score, were randomly assigned to $\operatorname{SCR}(n=116)$ or CAU $(n=107)$, using computer generated numbers. SCR-patients were invited for a Composite International Diagnostic Interview (CIDI) to diagnose depression and/or anxiety (interviewers were not blinded for group assignment). As part of the intervention, patients and their physicians were informed of the outcome of the CIDI in a letter and provided with treatment advice. At baseline and 6 month follow-up, depression and
\end{abstract}

\section{F. Pouwer $(\bowtie)$}

Centre of Research on Psychology in Somatic diseases (CoRPS),

Department of Medical Psychology \& Neuropsychology,

Tilburg University,

P.O. Box 90153, 5000 LE Tilburg, the Netherlands

e-mail: f.pouwer@uvt.nl

\section{F. Pouwer $\cdot$ F. J. Snoek}

Diabetes Psychology Research Group,

Department of Medical Psychology,

EMGO Institute, VU University Medical Centre,

Amsterdam, the Netherlands

\section{J. Tack}

Department of Internal Medicine,

Radboud University Nijmegen Medical Centre,

Nijmegen, the Netherlands diabetes-distress were measured using the Centre for Epidemiologic Studies Depression Scale (CES-D) and the Problem Areas in Diabetes survey (PAID). $\mathrm{HbA}_{1 \mathrm{c}}$ levels were obtained from medical charts.

Results Mean CES-D depression scores decreased from baseline to 6 months in both groups ( $24 \pm 8$ to $21 \pm 8$ [CAU] and $26 \pm 7$ to $22 \pm 10$ [SCR] respectively $[p<0.001]$ ), with no significant differences between groups. Neither diabetesdistress nor $\mathrm{HbA}_{1 \mathrm{c}}$ changed significantly within and between groups. The percentage of patients receiving mental healthcare increased in the SCR group from $20 \%$ to $28 \%$, compared with $15 \%$ to $18 \%$ in the CAU group. Conclusions/interpretation Depression screening with written feedback to patient and physician does not improve depression scores and has a limited impact on mental healthcare utilisation, compared with CAU. It appears that more intensive depression management is required to improve depression outcomes in patients with diabetes.

\section{P. H. L. M. Geelhoed-Duijvestijn}

Department of Internal Medicine, Haaglanden Medical Centre,

The Hague, the Netherlands

E. Bazelmans

Department of Medical Psychology,

Radboud University Nijmegen Medical Centre,

Nijmegen, the Netherlands

A. T. Beekman

Department of Psychiatry, EMGO Institute,

VU University Medical Centre,

Amsterdam, the Netherlands

R. J. Heine

Department of Endocrinology, Diabetes Centre,

VU University Medical Centre,

Amsterdam, the Netherlands 
Keywords Depression · Diabetes .

Randomised controlled trial $\cdot$ Screening

$\begin{array}{ll}\text { Abbreviations } \\ \text { CAU } & \begin{array}{l}\text { Care as usual } \\ \text { CES-D }\end{array} \\ & \begin{array}{l}\text { Centre for Epidemiologic Studies Depression } \\ \text { Scale }\end{array} \\ \text { CIDI } & \text { Composite International Diagnostic Interview } \\ \text { PAID } & \text { Problem Areas in Diabetes survey } \\ \text { SCR } & \text { Screening for depression }\end{array}$

\section{Introduction}

It has been shown that the prevalence of depression is relatively high in individuals with type 1 and type 2 diabetes, affecting $12-24 \%$ of patients [1,2]. Depression is not only associated with impaired quality of life [3], but also poorer glycaemic control, an increased risk for the development of diabetes complications and higher mortality rates [4-6]. Compared with non-diabetic controls, people with type 2 diabetes also have a $24 \%$ increased risk of developing depression [7]. The reasons for this increased prevalence and incidence of depression in diabetes are still poorly understood. The general view is that the burden of living with diabetes and its complications plays an important role in the aetiology of depression in diabetes $[8,9]$, as well as biochemical changes such as deregulation of the hypothalamic-pituitary-adrenal axis activities [10] and low-grade inflammation [11, 12].

Yet, it has been shown that depression in diabetes patients can be successfully treated by means of cognitive behavioural therapy, antidepressant medication, or a combination of the two. However, the study on the effectiveness of cognitive behavioural therapy in diabetes had considerable methodological limitations [13]. New intervention studies in this area are currently being conducted [14-16]. A crucial barrier to effective treatment is that recognition rates of depression are generally low. For example, in primary care, physicians failed to recognise depression in about $30-50 \%$ of their depressed patients [17]. In secondary care, diabetes nurse specialists failed to recognise and document high levels of anxiety, depression or diabetesspecific emotional distress in approximately $75 \%$ of cases who had established high scores on the Hospital Anxiety and Depression Scale (HADS) or Problem Areas in Diabetes (PAID) survey [18]. International clinical guidelines currently advocate screening for depression in patients with diabetes $[19,20]$. However, the effects of screening (case-finding) on psychological and diabetes outcomes have not yet been tested in a randomised controlled study in patients with diabetes [21]. Results from studies in patients without diabetes already suggest that screening for depression per se does not improve outcomes [22, 23]. Yet, Simon et al. [24] found a two-stage screening process as part of a stepped care depression treatment programme, delivered by specialised nurses, to be cost-effective. This finding would suggest that linking depression screening to depression treatment is an efficient approach.

The present study aimed to investigate whether active depression screening, using a stepped, mail-based screening procedure informing both patient and the treating physician, results in a significant decrease in depression scores compared with care as usual (CAU). Secondary outcomes investigated were: use of mental healthcare services, diabetes-distress and glycaemic control.

\section{Participants and methods}

Setting Data were collected in three tertiary diabetes clinics from different regions in the Netherlands: the VU University Medical Centre (Amsterdam), Radboud University Medical Centre (Nijmegen) and Haaglanden Medical Centre (The Hague). Each clinic serves a patient population of approximately 2,000 diabetes patients, both type 1 and type 2. A random sample of a total of 2,055 outpatients was drawn from the three respective clinics (Amsterdam $n=1,000$; (The Hague $n=555$, Nijmegen $n=500)$ ). Baseline data has been previously described in detail $[25,26]$.

Procedure At baseline, participants received questionnaire booklets by mail in two waves: the first assessing sociodemographics, clinical data and diabetes-specific distress. The second captured depression and mental healthcare consumption. We chose to separate the two booklets in order to avoid over-burdening the respondents. Patients were invited to return the questionnaires in pre-stamped envelopes to the research team. Inclusion criteria were: (1) adult ( $\geq 18$ years); (2) outpatient with established diabetes (type 1 or type 2); and (3) elevated depression score (Centre for Epidemiologic Studies Depression Scale (CES-D) score of 16 or more). Exclusion criteria were: (1) not being able to read Dutch; (2) a history of suicide attempt(s); (3) a history of hospital admission for depression; and (4) a history of electroconvulsive therapy (ECT) for depression. After completion of the depression questionnaire, respondents who met inclusion criteria were randomly assigned to: (1) CAU; or (2) the screening procedure (SCR). We randomised the participants using computer generated random numbers by SPSS. Allocation could not be completely concealed, due to the fact that physicians also received a copy of the letter regarding the outcome of the diagnostic psychiatric interview, and thus at least knew which participants were allocated to the intervention group. 
In the Netherlands, standard outpatient diabetes care generally consists of, on average, four regular appointments with an internist and/or diabetes nurse specialist. If needed, other members of the diabetes team can be consulted, such as a dietitian, podiatrist, ophthalmologist or medical psychologist [27]. It is important to emphasise that in the control group (CAU) the assessment of emotional wellbeing was not followed by feedback to patients and/or healthcare providers. Patients in the SCR group were invited for a Composite International Diagnostic Interview (CIDI), using the sections: Mood Disorders and Anxiety Disorders, allowing for a Diagnostic and Statistical Manual for Mental Disorders (DSM-IV) diagnosis [28]. In the case of a diagnosis of a mood disorder and/or an anxiety disorder, the patient, as well as his/her diabetes specialist and general practitioner, received a letter with the DSM-IV diagnosis, and advice regarding treatment options for depression and/or anxiety. When no mood or anxiety disorder was detected, the patients also received a letter with the outcome of the interview and the advice to contact their physician in case symptoms of depression/anxiety should worsen, as minor depression is known to be an important risk factor for the development of major depression in diabetes [29]. All patients received a brochure developed by mental health professionals and patients, supported by the Dutch Psychological Health Foundation (Fonds Psychische Gezondheid), about depression and its treatment. This brochure contains seven pages of information about the symptoms, the potential causes and the prevalence of depression. It also stresses the importance of seeking treatment, for example cognitive behavioural therapy or use of antidepressant medication. Finally, the brochure provides several useful addresses/phone numbers where patients can acquire further information and suggestions for self-help books (see www.psychischegezondheid.nl/dynamic/media/1/ files/depressie.pdf for the brochure [in Dutch].

At the 6 month follow-up, depression and diabetesdistress were reassessed by means of a mail questionnaire. Written informed consent was obtained from all participants and the study was approved by the local medical ethics advisory committee. The investigations were carried out in accordance with the principles of the Declaration of Helsinki as revised in 2000. The present study was not registered as a clinical trial, as the data collection in the three clinics took place between 2003 and 2007 and thus started before the prospective trial registries commenced (2005).

Demographic and clinical data This first questionnaire contained questions on: being single/having a partner, ethnic background (native Dutch, Turkish, Moroccan, or from Surinam, the Netherlands Antilles, Indonesia, or other), highest level of completed education, alcohol consumption and smoking. From the medical records of the patients, the following data were extracted: age, sex, type of diabetes, duration of diabetes, microvascular complications (retinopathy: background or proliferative, nephropathy and neuropathy), cardiovascular disease, most recent $\mathrm{HbA}_{1 \mathrm{c}}$ and blood pressure.

Assessment of depression To assess symptoms of depression, the Dutch version of the CES-D scale was used [30, 31]. This is a 20-item, self-report scale that asks respondents to indicate the frequency of occurrence of 20 depressive symptoms during the previous week. The instrument uses a four-point response set, ranging from 'rarely or none of the time' to 'most of the time or always'. Higher scores indicate more depressive symptoms and a cut-off point of 16 or more is generally accepted as indicative of a clinically significant level of depression symptoms [30, 31]. The automated World Health Organization CIDI-auto is a structured diagnostic interview, which was used to determine whether the patients suffered from a depressive disorder and/or an anxiety disorder, according to DSM-IV criteria [28, 32, 33]. The main advantage of the CIDI-auto is that the questions are fully specified. In our study, lay interviewers, mostly medical or psychology masters students, were trained by a certified CIDI interviewer (FP) to use the CIDI-auto. These interviewers were not blinded to group assignment.

Assessment of diabetes-specific emotional distress Diabetesspecific emotional distress was assessed using the Dutch version of the PAID survey $[34,35]$. This questionnaire consists of 20 items, which can be rated on a five-point Likert scale ranging from 0 (no problem) to 4 (serious problem). Examples of items are: 'Not accepting diabetes', 'Worrying about the future and the possibility of serious complications', 'Feeling overwhelmed by your diabetes regimen' or 'Feeling alone with diabetes'. Following the recommendation of the measure's authors, the PAID questionnaire scores are transformed to a scale of $0-100$, higher scores indicating more serious emotional problems.

Statistical analyses Statistical analyses were performed using SPSS version 16.0 (SPSS Inc., Chicago, IL, USA). The primary outcome variable was depression score; diabetes-distress, mental healthcare use and $\mathrm{HbA}_{1 \mathrm{c}}$ were regarded as secondary outcomes. The present study has sufficient power to detect a medium effect size of $f=0.25$. For example, the correlation between two time measures of CES-D depression score was assumed to be moderately large $r(\mathrm{~m} 1, \mathrm{~m} 2)=0.50$. Moreover, with a population standard deviation of the CES-D (each group) equalling 8 , the present study was able to detect a difference of four points in CES-D change scores between the two treatment groups with a power of 0.93 . The CES-D depression scores 
can range from 0 to 60 as, for each of the 20 items, the scores range from 0 to 3 .

Continuous demographic data for the participants were analysed using independent means $t$ tests, and $\chi^{2}$ tests were used for categorical variables. A $p$ value $<0.05$ was considered to be statistically significant. Data were analysed according to the intention-to-treat principle with the baseline value carried forward in cases of missing followup data. First, by means of $t$ tests and $\chi^{2}$ tests, baseline variables were compared between the intervention and CAU group. Then, general linear model repeated measures were conducted with the CES-D as dependent variable, with two independent variables: time (within-participant) and group (between-participant). The same analysis was performed with the PAID scores or $\mathrm{HbA}_{1 \mathrm{c}}$ as dependent variable.

\section{Results}

A total of 80 patients declined to participate, and 1,043 did not respond (Fig. 1), leaving 932 patients who returned the first questionnaire; 730 returned the second (depression) questionnaire. A history of suicide attempts was reason to exclude 58 patients, and another 507 patients were excluded because they had a CES-D depression score below 16. Six patients were excluded because they had received more than one inpatient treatment for depression. In total, 223 patients met inclusion criteria: 107 were randomly assigned to the CAU group and 116 to the SCR group, which included the CIDI with written feedback regarding the outcome of the interview (Fig. 1).

At baseline, the mean age of the 223 participants was 54 years, and almost $60 \%$ were women. Mean $\mathrm{HbA}_{1 \mathrm{c}}$ was $8.0 \%$ in both groups, mean BMI was 29 and $33-40 \%$ of the participants had type 1 diabetes. Of the patients with type 2 diabetes, $48-53 \%$ were treated with insulin. The majority of patients had a Europid ethnic background. A minority of respondents had a Surinamese $(6-7 \%)$ or Turkish ethnic background (3-5\%). Mean duration of diabetes (type 1 and type 2 taken together) was 17 years in both groups. At baseline, about a quarter of the patients had nephropathy, cardiovascular disease and/or background retinopathy. Neuropathy was present in $26-35 \%$ of the patients. Sociodemographic and clinical variables did not differ significantly between the intervention and the control group
Fig. 1 Screening for depression in outpatients with diabetes study: patient enrolment

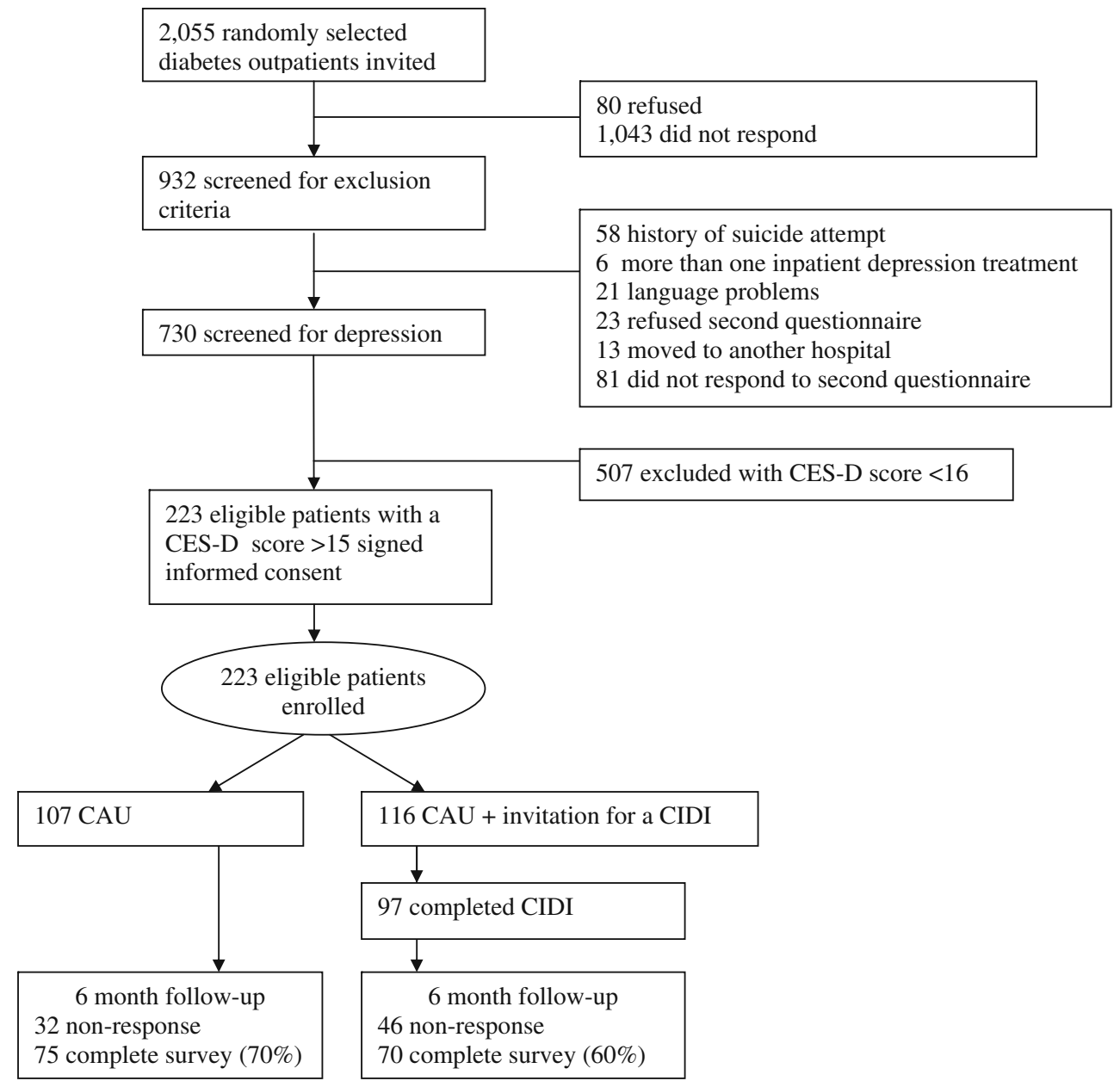


(Table 1). Patients in the CAU group more often reported low education ( $43 \%$ vs $31 \%$ in the intervention group, NS). In an additional analysis we compared baseline characteristics of those who were lost to follow-up with those who completed the study and found the drop outs had higher baseline depression scores than completers $(27 \pm 8$ vs $24 \pm 7$, $p<0.003)$, more often had low education ( $46 \%$ vs $32 \%$, $p=0.03$ ) and more often had a non-Dutch ethnic background ( $25 \%$ vs $9 \%, p=0.002)$. The groups did not differ regarding age, BMI, type of diabetes or baseline $\mathrm{HbA}_{1 \mathrm{c}}$.

Prevalence of mood and anxiety disorders in the intervention group Data on the prevalence of mood and anxiety disorders in the intervention group, based on the results of the CIDI interviews, have been published elsewhere [23]. In brief, $8 \%$ of the patients with type 1 diabetes were diagnosed with a depressive disorder (no sex difference), compared with $2 \%$ of men and $21 \%$ of women with type 2 diabetes. In the present study, 116 patients with a high CES-D score were invited for a CIDI interview: 13 refused to be interviewed and 6 could not be contacted. Thus 97 patients underwent the psychiatric diagnostic interview. In the intervention group, 54\% (52/97) of those invited suffered subthreshold depression (no mood or anxiety disorder diagnosed). Thirty-two patients were diagnosed with a mood disorder (33\%), 14 patients with generalised anxiety disorder (15\%), one with blood/injection phobia $(1 \%)$, six patients with natural/environment phobia $(6 \%)$, 15 with social phobia $(16 \%), 3$ with panic disorder $(3 \%)$ and five patients with agoraphobia (5\%). Twenty-eight patients $(30 \%)$ were diagnosed with two or more mood/ anxiety disorders.
Table 1 Baseline demographic and clinical characteristics of intervention and CAU patients
SBP, systolic blood pressure; DBP, diastolic blood pressure

\begin{tabular}{|c|c|c|c|}
\hline \multirow[b]{2}{*}{$n$} & \multirow{2}{*}{$\frac{\text { SCR }}{116}$} & \multirow{2}{*}{$\frac{\text { CAU }}{107}$} & \multirow[t]{2}{*}{$p$ value } \\
\hline & & & \\
\hline \multicolumn{4}{|l|}{ Demographics } \\
\hline Age (years) & $55 \pm 12$ & $52 \pm 16$ & 0.150 \\
\hline No partner $(\%)$ & $32(37 / 116)$ & $28(30 / 107)$ & 0.561 \\
\hline Low education $(\%)$ & $43(50 / 116)$ & $31(33 / 107)$ & 0.072 \\
\hline Female sex $(\%)$ & $58(67 / 116)$ & $58(62 / 107)$ & 0.978 \\
\hline \multicolumn{4}{|l|}{ Ethnicity } \\
\hline Dutch $(\%)$ & $87(94 / 108)$ & $81(81 / 100)$ & \multirow[t]{5}{*}{0.734} \\
\hline Moroccan (\%) & $2(2 / 108)$ & $1(1 / 100)$ & \\
\hline Turkish (\%) & $3(3 / 108)$ & $5(5 / 100)$ & \\
\hline Surinami $(\%)$ & $7(7 / 108)$ & $6(6 / 100)$ & \\
\hline Other $(\%)$ & $1(3 / 108)$ & $6(6 / 100)$ & \\
\hline \multicolumn{4}{|l|}{ Hospital } \\
\hline VUmc, Amsterdam (\%) & $48(56 / 116)$ & $47(50 / 107)$ & \multirow[t]{3}{*}{0.967} \\
\hline Westeinde, The Hague (\%) & $28(32 / 116)$ & $29(31 / 107)$ & \\
\hline St Radboud, Nijmegen (\%) & $24(28 / 116)$ & $24(26 / 107)$ & \\
\hline \multicolumn{4}{|l|}{ Clinical values } \\
\hline $\mathrm{HbA}_{1 \mathrm{c}}(\%)$ & $8.0 \pm 1.4$ & $8.0 \pm 1.5$ & 0.669 \\
\hline BMI & $29 \pm 7$ & $29 \pm 8$ & 0.787 \\
\hline $\mathrm{SBP}(\mathrm{mmHg})$ & $138 \pm 16$ & $136 \pm 18$ & 0.508 \\
\hline $\mathrm{DBP}(\mathrm{mmHg})$ & $77 \pm 10$ & $76 \pm 10$ & 0.498 \\
\hline Type 1 diabetes $(\%)$ & $33(38 / 116)$ & $40(43 / 107)$ & \multirow[t]{3}{*}{0.515} \\
\hline Type 2 diabetes (diet/oral hypoglycaemic agents) (\%) & $14(16 / 116)$ & $12(13 / 107)$ & \\
\hline Type 2 diabetes treated with insulin (\%) & $53(62 / 116)$ & $48(51 / 107)$ & \\
\hline Nephropathy (\%) & $20(20 / 102)$ & $30(29 / 97)$ & \multirow[t]{2}{*}{0.092} \\
\hline Background retinopathy (\%) & $28(29 / 103)$ & $20(19 / 95)$ & \\
\hline Proliferative retinopathy $(\%)$ & $5(5 / 103)$ & $7(7 / 95)$ & 0.350 \\
\hline Neuropathy $(\%)$ & $35(35 / 101)$ & $26(25 / 96)$ & 0.189 \\
\hline Any microvascular disease $(\%)$ & $65(67 / 103)$ & $51(49 / 97)$ & 0.033 \\
\hline Cardiovascular disease $(\%)$ & $26(26 / 100)$ & $25(25 / 93)$ & 0.890 \\
\hline Duration of diabetes (years) & $17 \pm 11$ & $17 \pm 11$ & 0.954 \\
\hline CES-D depression score & $26 \pm 7$ & $24 \pm 7$ & 0.199 \\
\hline
\end{tabular}


Effects of the screening intervention on mental healthcare consumption The percentage of patients at baseline reporting to have received (any) psychological treatment in the past did not differ between the two groups (41\% [42/103] in the CAU group vs $44 \%$ [50/114] in the SCR group, $p=0.14$ ). At baseline, the percentages of patients that reported being under treatment for depression did not differ significantly ( $15 \%$ in the CAU and $20 \%$ in the SCR group). The percentage of patients that reported to have received depression treatment during the study period did not differ significantly between the groups $(18 \%$ in the CAU and $28 \%$ in the SCR group).

Effects of the screening intervention on depression score, diabetes-distress and $\mathrm{HbA}_{1 c}$ Follow-up data was complete for 145 of 223 patients $(65 \%)$. At the 6 month follow-up, depression scores had significantly improved in both depressed groups $(p<0.001)$, but the improvement did not differ between groups ( $21 \pm 8$ [CAU] vs $22 \pm 10$ [SCR], NS). Although mean CES-D scores had decreased after 6 months, the percentage of patients with elevated depression scores (CES-D $\geq 16$ ) was still relatively high and did not differ between groups $(68 \%$ [CAU] vs $75 \%$ [SCR], NS).

General linear model repeated measures analysis confirmed that the screening intervention did not affect CES-D depression scores nor the level of diabetes-specific emotional distress. In the analysis using CES-D, there was a significant time effect $(p<0.001)$, with both groups showing a decrease in depression scores, but no significant group effect over time. Intention-to-treat analysis with the baseline value carried forward in the case of missing follow-up data, confirmed the absence of effects of the screening intervention on depression scores or levels of diabetes-specific distress, or $\mathrm{HbA}_{1 \mathrm{c}}$ (data not shown).

\section{Discussion}

The main finding of the present study is that a mail-based screening procedure for depression in outpatients with diabetes did not have a substantial impact on the number of patients seeking mental healthcare. This could explain why the screening procedure did not yield better outcomes compared with CAU with respect to CES-D depression scores, diabetes-distress or $\mathrm{HbA}_{1 \mathrm{c}}$. Importantly, levels of depression, anxiety and diabetes-specific distress remained relatively high in both groups.

The results of the present study are in line with earlier studies that have been carried out in primary care settings, showing that disclosure of depression status in itself, based on case-finding instruments, does not impact depression scores [21, 36-38]. Apparently, detection of depression does not automatically prompt physicians or patients to seek mental healthcare. In our study, letters were sent to the depressed patients and their treating physicians. Although this intervention included advice to seek treatment for depression, this was not tied to a specific treatment programme nor was there any follow-up to check if action had been taken. Simon et al. [24] have shown that linking the screening outcome to treatment does help to achieve better depression outcomes. Indeed, in an earlier trial testing the effects of individual monitoring and discussing emotional well-being as part of the periodic diabetes consultation in secondary care by diabetes nurse specialists, we found significant improvements in emotional well-being compared with CAU [39]. We should, therefore, not only screen for depression in diabetes care, as advocated by the ADA and IDF [19, 20], but ensure that the depression screening outcome is combined with an appropriate depression treatment programme [21, 40].

Strengths of our study include its multicentre randomised controlled design, the use of a diagnostic interview schedule that allows diagnosis of depressive disorder and the relatively large sample size. The present study has sufficient power to detect a medium effect size of $f=0.25$. On the other hand, response was rather low and selection bias during the inclusion phase and selective loss to followup might have influenced the results. As depressed patients often lack energy and optimism, it may be that a relatively large number of the depressed patients did not return the questionnaire at baseline. In addition, later in the study, a considerable number of male participants with type 2 diabetes refused the psychiatric diagnostic interview. In our study, loss to follow-up was particularly associated with a high baseline depression score, a low level of education and non-Dutch descent. Attrition bias may have led to underestimation of the effect. However, unfortunately, it is not possible to check this assumption.

Overall, the percentage of male participants is lower than would be expected. Particularly, male participants with a non-Dutch background seem to be under-represented. This may have influenced our results. However, the screening procedure appeared to be feasible and generally acceptable to patients, with only a few refusals.

Moreover, we compared CAU (with no diagnostic interview) with a complex intervention consisting of several steps: (1) assessment of depression using a self-report questionnaire; (2) a diagnostic psychiatric interview in case of a high depression score; (3) feedback regarding the outcome of the diagnostic interview to patient, internist and primary care physician; and (4) brochure on depression. An inevitable limitation of our design is that we were not able to determine the exact impact of each separate step, such as the diagnostic interview.

The question arises as to the clinical implications of our findings. It would seem appropriate to revisit the recom- 
mendations of the ADA, IDF and other diabetes organisations to screen for depression, and stress the importance of linking screening to treatment. For this purpose, mental health services for diabetes patients need to be in place. Unfortunately, psychological services for diabetes patients are still lacking or scarce in most settings [41, 42]. This calls for improving access to mental health services in diabetes as well as developing new therapeutic strategies that can enlarge reach at relatively low costs, for example by offering web-based programmes [14].

In summary, our study shows that a mail-based screening procedure for depression in outpatients with diabetes only marginally affects uptake of mental healthcare, thereby failing to improve depression scores, levels of diabetesspecific emotional stress or glycaemic control. It is imperative that screening for depression is embedded in a comprehensive depression treatment programme.

Acknowledgements The authors would like to express their gratitude towards the patients who participated in this study.

Funding Dutch Diabetes Research Foundation (Diabetesfonds), 2000.00 .018 .

Duality of interest R. J. Heine is currently employed by Eli Lilly and owns shares. The other authors declare that they have no duality of interest associated with this manuscript.

Open Access This article is distributed under the terms of the Creative Commons Attribution Noncommercial License which permits any noncommercial use, distribution, and reproduction in any medium, provided the original author(s) and source are credited.

\section{References}

1. Barnard KD, Skinner TC, Peveler R (2006) The prevalence of comorbid depression in adults with type 1 diabetes: systematic literature review. Diabet Med 23:445-448

2. Ali S, Stone MA, Peters JL, Davies MJ, Khunti K (2006) The prevalence of co-morbid depression in adults with type 2 diabetes: a systematic review and meta-analysis. Diabet Med 23:1165-1173

3. Schram M, Baan CA, Pouwer F (2009) Depression and quality of life in patients with diabetes: a systematic review from the European Depression in Diabetes (EDID) Research Consortium. Curr Diab Rev 5:112-119

4. Lustman PJ, Anderson RJ, Freedland KE, de Groot M, Carney RM, Clouse RE (2000) Depression and poor glycemic control: a meta-analytic review of the literature. Diab Care 23:934-942

5. Ismail K, Winkley K, Stahl D, Chalder T, Edmonds M (2007) A cohort study of people with diabetes and their first foot ulcer: the role of depression on mortality. Diab Care 30:1473-1479

6. Egede LE, Nietert PJ, Zheng D (2005) Depression and all-cause and coronary heart disease mortality among adults with and without diabetes. Diab Care 28:1339-1345

7. Nouwen A, Winkley K, Twisk J, for the European Depression in Diabetes (EDID) Research Consortium et al (2010) Type 2 diabetes mellitus as a risk factor for the onset of depression: a systematic review and meta-analysis. Diabetologia 53:2480 2486

8. de Groot M, Anderson R, Freedland KE, Clouse RE, Lustman PJ (2001) Association of depression and diabetes complications: a meta-analysis. Psychosom Med 63:619-630

9. Pouwer F, Beekman AT, Nijpels G et al (2003) Rates and risks for co-morbid depression in patients with type 2 diabetes mellitus: results from a community-based study. Diabetologia 46:892-898

10. Penninx BW, Beekman AT, Bandinelli S et al (2007) Late-life depressive symptoms are associated with both hyperactivity and hypoactivity of the hypothalamo-pituitary-adrenal axis. Am J Geriatr Psychiatry 15:522-529

11. Pickup JC (2004) Inflammation and activated innate immunity in the pathogenesis of type 2 diabetes. Diab Care 27:813-823

12. Dantzer R, O'Connor JC, Johnson FGG, RW KKW (2008) From inflammation to sickness and depression: when the immune system subjugates the brain. Nat Rev Neurosci 9:46-57

13. van der Feltz-Cornelis CM, Nuyen J, Stoop C et al (2010) Effect of interventions for major depressive disorder and significant depressive symptoms in patients with diabetes mellitus: a systematic review and meta-analysis. Gen Hosp Psychiatry 32: 380-395

14. van Bastelaar KM, Pouwer F, Cuijpers P, Twisk JW, Snoek FJ (2008) Web-based cognitive behavioural therapy (W-CBT) for diabetes patients with co-morbid depression: design of a randomised controlled trial. BMC Psychiatry 8:9

15. Petrak F, Hautzinger M, Plack K et al (2010) Cognitive behavioural therapy in elderly type 2 diabetes patients with minor depression or mild major depression: study protocol of a randomized controlled trial (MIND-DIA). BMC Geriatr 10:21

16. Katon W, Lin EH, Von Korff M et al (2010) Integrating depression and chronic disease care among patients with diabetes and/or coronary heart disease: the design of the TEAMcare Study. Contemp Clin Trials 31:312-322

17. Tylee A, Walters P (2007) Underrecognition of anxiety and mood disorders in primary care: why does the problem exist and what can be done? J Clin Psychiatry 68(Suppl 2):S27-S30

18. Pouwer F, Beekman AT, Lubach C, Snoek FJ (2006) Nurses' recognition and registration of depression, anxiety and diabetesspecific emotional problems in outpatients with diabetes mellitus. Patient Educ Couns 60:235-240

19. American Diabetes Association (2009) Standards of medical care in diabetes - 2009. Diab Care 32(Suppl 1):S13-S61

20. International Diabetes Federation (2005) Global guideline for type 2 diabetes. International Diabetes Federation. Available from www.idf.org/Global_guideline, accessed 9 June 2010

21. Pouwer F (2009) Should we screen for emotional distress in type 2 diabetes mellitus? Nat Rev Endocrinol 5:665-671

22. Gilbody S, Sheldon T, House A (2008) Screening and casefinding instruments for depression: a meta-analysis. CMAJ 178:997-1003

23. Thombs BD, de Jonge P, Coyne JC et al (2008) Depression screening and patient outcomes in cardiovascular care: a systematic review. JAMA 300:2161-2171

24. Simon GE, Katon WJ, Lin EH et al (2007) Cost-effectiveness of systematic depression treatment among people with diabetes mellitus. Arch Gen Psychiatry 64:65-72

25. Pouwer F, Geelhoed-Duijvestein PH, Tack CJ et al (2010) Prevalence of co-morbid depression is high in outpatients with type 1 or type 2 diabetes mellitus. Results from three outpatient clinics in the Netherlands. Diabet Med 27:217-224

26. van Bastelaar KMP, Pouwer F, Geelhoed-Duijvestijn PH et al (2010) Diabetes distress mediates the association between depressive symptoms and glycaemic control in type 1 and type 2 diabetes. Diabet Med 27:798-803 
27. Bakker K, Bilo HJG (2004) Diabetes care in the Netherlands: now and in the future. Pract Diabetes Int 21:88-91

28. Andrews G, Peters L (1998) The psychometric properties of the Composite International Diagnostic Interview. Soc Psychiatry Psychiatr Epidemiol 33:80-88

29. Bot M, Pouwer F, Ormel J, Slaets JPJ, De Jonge P (2010) Predictors of incident major depression in diabetic outpatients with subthreshold depression. Diabet Med 27:1295-1301

30. Radloff LS (1977) The CES-D scale: a self-report depression scale for research in the general population. Appl Psychol Meas 1:385-401

31. Beekman AT, Deeg DJ, van Limbeek J, Braam AW, De Vries MZ, van Tilburg W (1997) Criterion validity of the Center for Epidemiologic Studies Depression scale (CES-D): results from a community-based sample of older subjects in the Netherlands. Psychol Med 27:231-235

32. Wittchen HU (1994) Reliability and validity studies of the WHOComposite International Diagnostic Interview (CIDI): a critical review. J Psychiatr Res 28:57-84

33. American Psychiatric Association (1994) Diagnostic and statistical manual of mental disorders, 4th edn. American Psychiatric Association, Washington

34. Welch GW, Jacobson AM, Polonsky WH (1997) The problem areas in diabetes scale. An evaluation of its clinical utility. Diabetes Care 20:760-766

35. Snoek FJ, Pouwer F, Welch GW, Polonsky WH (2000) Diabetesrelated emotional distress in Dutch and U.S. diabetic patients: cross-cultural validity of the problem areas in diabetes scale. Diab Care 23:1305-1309

36. Dowrick C, Buchan I (1995) Twelve month outcome of depression in general practice: does detection or disclosure make a difference? BMJ 311:1274-1276

37. Whooley MA, Stone B, Soghikian K (2000) Randomized trial of case-finding for depression in elderly primary care patients. J Gen Intern Med 15:293-300

38. Lewis G, Sharp D, Bartholomew J, Pelosi AJ (1996) Computerized assessment of common mental disorders in primary care: effect on clinical outcome. Fam Pract 13:120-126

39. Pouwer F, Snoek FJ, van der Ploeg HM, Adèr HJ, Heine RJ (2001) Monitoring of psychological well-being in outpatients with diabetes: effects on mood, $\mathrm{HbA}(1 \mathrm{c})$, and the patient's evaluation of the quality of diabetes care: a randomized controlled trial. Diab Care 24:1929-1935

40. Katon WJ, Von Korff M, Lin EH et al (2004) The Pathways Study: a randomized trial of collaborative care in patients with diabetes and depression. Arch Gen Psychiatry 61:1042-1049

41. Nicholson TR, Taylor JP, Gosden C, Trigwell P, Ismail K (2009) National guidelines for psychological care in diabetes: how mindful have we been? Diabet Med 26:447-450

42. Peyrot M, Rubin RR, Siminerio LM (2006) Physician and nurse use of psychosocial strategies in diabetes care: results of the crossnational Diabetes Attitudes, Wishes and Needs (DAWN) Study. Diab Care 29:1256-1262 